\title{
Bimolecular Photophysics
}

\author{
David L. Andrews and Philip Allcock \\ School of Chemical Sciences, University of East Anglia, Norwich NR4 7TJ, U.K.
}

\section{Introduction}

There is a wide range of photophysical processes entailing pairwise interaction between molecules. Whilst many specifically chemical interactions occur between molecules close enough for their electronic orbitals to overlap, as for example in bimolecular reactions and collision processes in general, there are many other types that occur through electromagnetic coupling at appreciably larger distances. For example the migration of energy between molecules, a highly significant feature of ultrafast photochemistry in the condensed phase, generally occurs beyond the subnanometer distances where direct energy exchange could result from wavefunction overlap, because the individual species involved are too far apart. The dominant processes for resonant energy transfer are then radiative transfer, in which photons are emitted by excited molecules and subsequently absorbed by others, and a 'radiationless' interaction. Both of these processes play a significant role in the dynamics of energy trapping in the photosynthetic unit. Indeed the high efficiency of photosynthesis in green plants is substantially attributable to the speed with which energy migrates from 'antenna' chlorophyll molecules to reaction centres where the chemistry occurs. In a quite different area, we find essentially the same process playing a significant role in determining the performance characteristics of laser crystals. Many other photoinduced processes involving intermolecular coupling are increasingly the subject of research interest; examples include fluorescence migration, induced circular dichroism, and cooperative absorption. In this review these and other processes are discussed with reference to a new formulation of theory embracing both radiative and 'radiationless' energy transfer.

At the outset, it may be helpful to identify more precisely the unifying theme of the various photophysical topics to be addressed. Figure 1 shows in a greatly simplified form the essential details: (a) depicts the simple case of energy transfer between two molecules, where no radiation either impinges on, or is emitted by, either one of the pair. Of course radiation will play a part by initially providing the excitation that is shown passing from one molecule to another, but it plays no intrinsic part in the transfer itself. Figure 1(b) shows a process with the next degree of complexity, specifically involving the absorption of a photon by one of the participating molecules. Such pro-

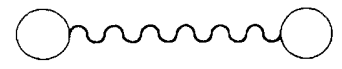

(a)

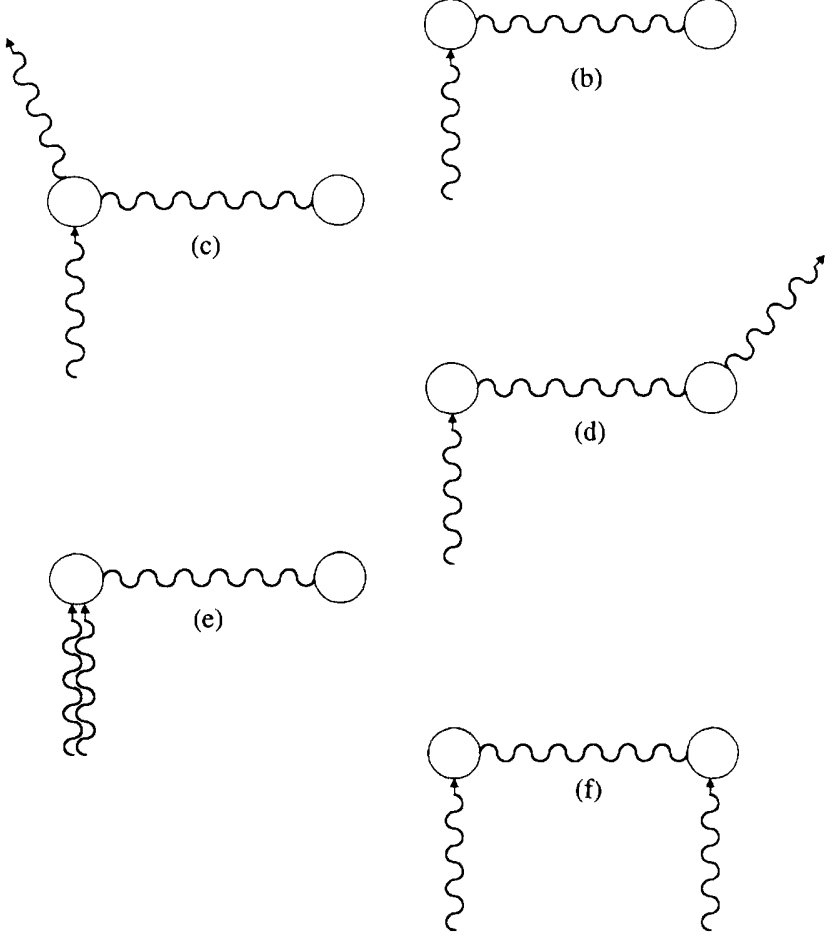

Figure 1 Simple representations of bimolecular processes involving molecules A and B (left and right, respectively, in each pair). Here and in subsequent figures, applied radiation is shown incident from below and emergent radiation above the pair, the horizontal wave representing the coupling.

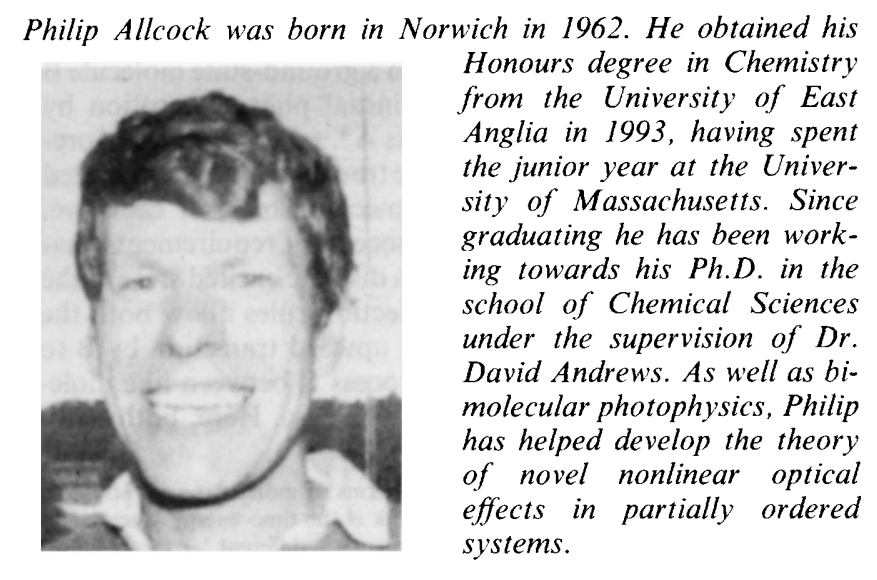

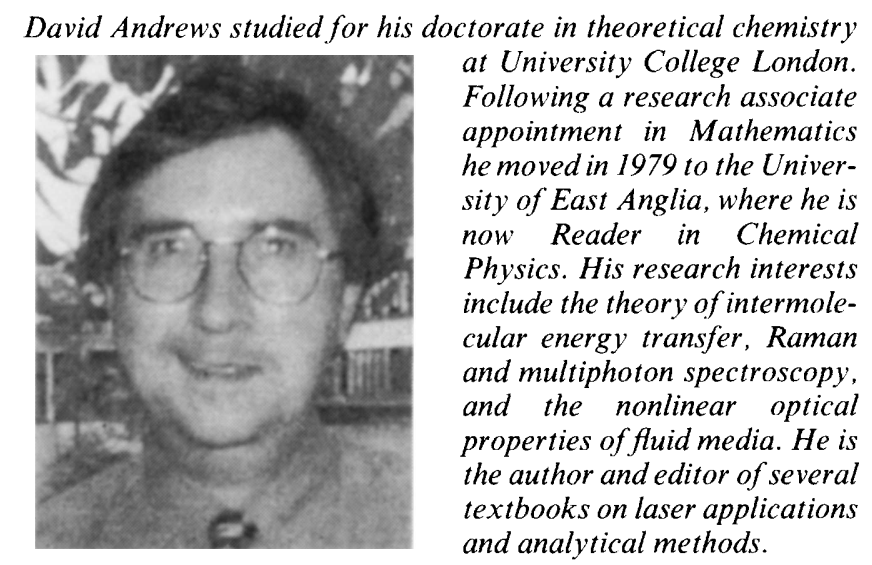


cesses are often manifest through changes in the optical characteristics of one chemical species under the influence of another, as in induced absorption and induced circular dichroism Another illustration is afforded by cooperative or sum-frequency excitation More complex cases to be considered are those in which two photons are involved Figures $1(\mathrm{c})$ and (d) both represent phenomena where photon energy both enters and leaves the bimolecular system, one important case being the fluorescence migration employed to characterize protein structures, another less well-known member of the same class is sequential Raman scattering Finally, there are bimolecular two-photon absorption processes, as depicted in Figures 1(e) and ( $\mathrm{f}$ ), associated inter alla with trans-interface absorption and anomalies in spectra based on broadband radiation As each process is examined in turn, our attention will focus on their distınctive aspects, primarily concernıng the selection rules and dependence on molecular separation For those familiar with the concepts of dipole coupling a general basis of theory is first outlined in Section 2, other readers may continue at Section 3

\section{Intermolecular Coupling}

At the beginning it is appropriate to address the detalled nature of the coupling that mediates the various bimolecular processes to be considered The description to be employed is one that proves to accommodate not only coupling associated with intermolecular energy transfer, but also the interactions between polar molecules, $t$ e static coupling between permanent dipole moments As the latter is more familiar, it is helpful to use it as a starting point

Consider the energy of interaction between two polar molecules $\mathrm{A}$ and $\mathrm{B}$, described by the familiar formula,

$$
M=\frac{1}{4 \pi \epsilon_{0} R^{3}}\left\{\left(\mu_{\mathrm{A}} \cdot \mu_{\mathrm{B}}\right)-3\left(\mu_{\mathrm{A}} \cdot \boldsymbol{R}\right)\left(\mu_{\mathrm{B}} \cdot \hat{R}\right)\right\},
$$

where $\mu_{\mathrm{A}}$ and $\mu_{\mathrm{B}}$ are the (permanent) dipole moments of the two molecules, and $\hat{R}$ is the unit vector of molecular separation Although unusual, it is helpful to consider the role of the timeenergy uncertainty principle in such an interaction Since no energy is transferred between the molecules, there is clearly no energy uncertainty consequently the interaction formula (1) applies over an unrestricted range of separation distances, $R$ and times $t=R / c$, where $c$ is the velocity of hight From the inverse cubic dependence on $R$ in equation 1 it is evident that the fields experienced by an interacting pair fall off very rapidly with increasing molecular separation

For the photophysical processes that will primarily concern us in this review, generally characterized by the fact that both molecules undergo a change in electronic or vibrational state, it is rates or corresponding intensities rather than energies that require evaluation The transitions involved, as energy is transferred from one molecule to another, necessarily entail transition dipole coupling, and so can be displayed by both polar and nonpolar molecules The appropriate rate equations are derived from the standard methods of time-dependent perturbation theory through the Fermı Golden Rule ${ }^{1}$ shown explicitly in equation 2

$$
\Gamma_{\mathrm{fi}}=\frac{2 \pi}{\hbar}\left|M_{\mathrm{fi}}\right|^{2} \delta\left(E_{\mathrm{f}}\right)
$$

Here $\Gamma_{\mathrm{fi}}$ is the rate of transition from an initial system state 1 to a final state $\mathrm{f}, M_{\mathrm{f}}$ is the quantum probability amplitude for the process - a transitional analogue of the $M$ in equation 1 - and the Dirac delta function represents overall conservation of energy, in the sense that there is no gain or loss of energy by the system comprising the molecules and the radiation

The derivation of a result for $M_{\mathrm{fi}}$ that is appropriate for both radiatıve and non-radiative energy transfer interactions, outside the region of wavefunction overlap, is one of the distinctive recent successes of Quantum Electrodynamics (QED) Retarda- tion features in the transfer process, associated with the finite tıme for signal propagation between molecules, were first revealed in pioneering works almost thirty years ago ${ }^{23}$ Although no differentiation was initially made between the representations of short-range non-radiative and long-range radiative transfer, their specific consideration as asymptotic limits of a more general unified mechanısm over an unrestricted range of distances $R$ is more recent ${ }^{4}$ The QED formulation of energy transfer between molecules is naturally cast in terms of quantized field particles (virtual photons), and as such naturally accommodates the retardation effects which modify the inverse power law governing distance dependence ${ }^{5}{ }^{8}$ Similar features arise elsewhere, for example in correctly modifying at large distances the $R{ }^{6}$ dependence of the London potential (the attractive part of the familiar Lennard-Jones interaction) to a long-range $R{ }^{7}$ behaviour as given by the Casimir-Polder formula ${ }^{9}$ The QED formulation in which the two energy transfer mechanisms are united is attractive in comparison to the traditional dipole-dipole coupling approach, not least in establishıng the lack of any competition between radiative and radiationless coupling mechanisms

From the unified theory, it emerges that the explicit result for $M_{\mathrm{fi}}$, for a process entalling transfer of an energy $h \nu=h c k$ from A to $\mathrm{B}$, is expressible as

$$
\begin{aligned}
M_{\mathrm{f}}= & \frac{\exp (\imath k R)}{4 \pi \epsilon_{0} R^{3}}\left[(1-\imath k R)\left\{\left(\mu_{\mathrm{A}}^{\downarrow} \cdot \mu_{\mathrm{B}}^{\dagger}\right)-3\left(\mu_{\mathrm{A}}^{\downarrow} \cdot \hat{R}\right)\left(\mu_{\mathrm{B}}^{\dagger} \hat{\boldsymbol{R}}\right)\right\}\right. \\
& \left.-k^{2} R^{2}\left\{\left(\mu_{\mathrm{A}}^{\downarrow} \mu_{\mathrm{B}}^{\dagger}\right)-\left(\mu_{\mathrm{A}}^{\downarrow} \cdot \hat{R}\right)\left(\mu_{\mathrm{B}}^{\dagger} \cdot \hat{R}\right)\right\}\right]
\end{aligned}
$$

where the transition dipole moments of $\mathrm{A}$ and $\mathrm{B}$ are differentrated by arrows for the upward and downward transitions respectively Inspection of the uncertainty principle now offers a simple way of understanding the results in terms of the intermolecular distances ${ }^{\dagger}$ Here, because energy is transferred between molecules, the interaction formula clearly does not yield the same inverse power dependence on the separation $R$ for all tımes Over very short times, associated with small intermolecular distances where $k R \ll 1$, the transfer energy cannot be localized in either A or B and it remains the case that the coupling takes the form of equation 1, essentially reflectıng a static $\left(R^{3}\right)$ interaction However at relatively large tımes, corresponding to coupling over distances where $k R \gg 1$, the propagating character of the energy in transit becomes more evident, leading to radiative $\left(R^{-1}\right)$ behaviour

\section{Intermolecular Energy Transfer}

Having introduced intermolecular couphing processes as the link between various bimolecular photophysical interactions, we now consider the specific case of resonant energy transfer ${ }^{4} 810$ As noted earlier, this is a process that plays a major role in the primary events of photosynthesis - it is also a significant dynamical feature of many other phenomena such as chemiluminescence and laser emission

Let us suppose that molecule A is initially in an excited state and the process of interest concerns the ultrafast migration of energy across an arbitrary distance to a ground-state molecule B - we are not concerned with the initial photoabsorption by molecule $\mathrm{A}$, only the transfer process $\mathrm{A}^{*}+\mathrm{B} \rightarrow \mathrm{A}+\mathrm{B}^{*}$ Moreover we need not specify whether the transfer is to be considered radiationless (the familiar Forster mechanism ${ }^{11}$ ) or radiative, for reasons that will emerge The necessary requirements that the transfer energy is resonant with a discrete excited state of the recipient molecule, and that the selection rules allow both the downward transition by $\mathrm{A}$ and the upward transition by $\mathrm{B}$ to occur, suggests that the transfer process is between like molecules, though this is not necessarily the case Here, both path-

+ In formulating the limitıng behaviour in terms of molecular separation $R$ transition times $t$ and wave vector $k$ the link is the time-energy Uncertainty Principle expressed through equivalent different forms ds 


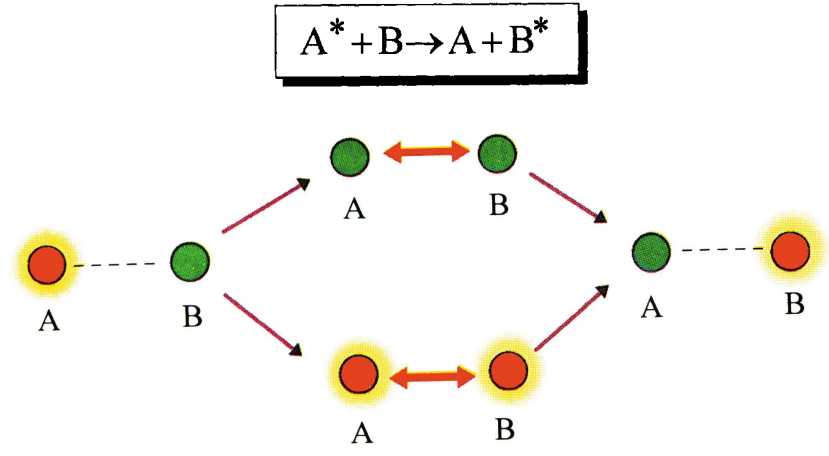

Figure 2 Two routes for intermolecular energy transfer, with differing intermediate states In the top route, excitation energy leaves A before being captured by $\mathbf{B}$, in the counterpart lower route both molecules simultaneously carry excitation energy The lower route is significant only for very short tımes

ways shown in Figure 2 have to be considered, in order to take account of the non-energy conserving route allowed by the Uncertainty Principle at very short tımes

The transfer rate $\Gamma_{\mathrm{f}_{1}}$ depends on the probability amplitude (equation 3) times its complex conjugate, and is concisely expressible as the following excitation transfer function,

$$
A(k, R) \sim R \quad\left[3+k^{2} R^{2}+k^{4} R^{4}\right],
$$

clarifying the dependence on the transfer energy hck and the molecular separation $R$ The form of equation 4 reveals not only the short-range Forster $R^{-6}$ and the long-range radiative $R^{2}$ behaviour, but also an intermediate dependence manifest in the $R^{-4}$ term, apparent at molecular separations where $k R \sim 1$ This is a direct result of the QED formulation that yields equation 3 The best way to see this influence on the energy transfer rate is in a $\log$ plot of the function $A(k, R)$ against $R$ This is illustrated in Figure 3 , where we assign to the wavevector magnitude $k$ the value $9 \times 10^{6} \mathrm{~m}^{1}$, corresponding to a transfer of energy following photoabsorption at $\sim 700 \mathrm{~nm}$ This gives an illustration of the range dependence of energy transfer between chlorophyll molecules within the photosynthetic unit (PSU)

Thus, it is the scale of the intermolecular separation that determınes whether a process is seen as either a radiative or nonradiative transfer - in fact both are limiting cases of a single unified mechanısm The subtle difference is that over very short intermolecular distances the photon is undetected, and hence commonly referred to as 'virtual', in the same sense as the virtual molecular states that arise for example in Raman scattering This links with the fact that at short time intervals the interaction

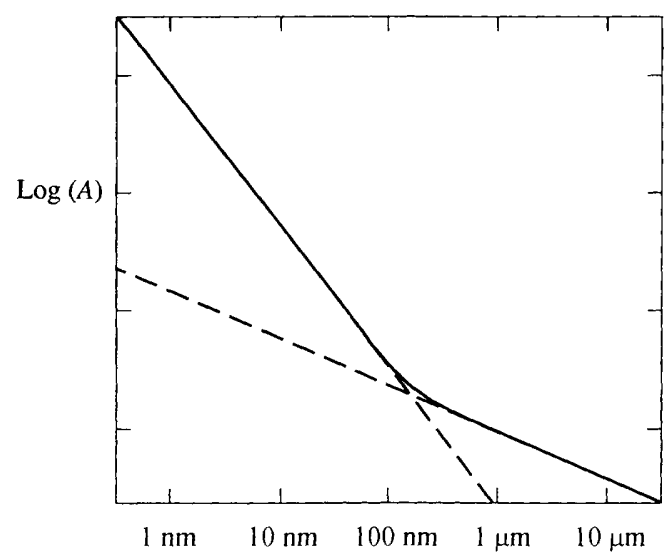

Figure 3 Logarithmic plot of the excitation transfer function $A(k, R)$ against intermolecular distance, with $k=9 \times 10^{6} \mathrm{~m}{ }^{1}$ Dotted lines indicate the asymptotic $R^{6}$ and $R^{2}$ behaviour at distances small and large, respectively, compared with $k^{1}$ is almost instantaneous, and as such the short range limit of equation 3 has the same character as the classical dipole-dipole interaction (equation 1)

\section{Induced Absorption and Circular Dichroism}

In considering intermolecular energy transfer, we noted that the preceding photoabsorption did not need to be taken into consideration as it constitutes a separate process, in other words the photon absorption and energy transfer represent two stages in a definite sequence Now we turn our attention to some rather different processes, in which coupling between close proximity molecular pairs plays a significant part in modifyıng the absorption process itself One key feature of the bimolecular interaction to be discussed below is a change in the selection rules of symmetric species Another aspect is the induced circular dichroism which may be apparent in the absorption spectrum of an achiral species through couplıng with a chiral partner

The first, and in a sense prototypical, example of bimolecular photoabsorption is a process in which a molecule $\mathrm{A}$ undergoes optical excitation under the dual influence of the radiation and a static interaction with a neighbouring polar molecule $B$ $\mathbf{A}+\mathbf{B}+h v \rightarrow \mathbf{A}^{*}+\mathbf{B}$, as shown in Figure 4 In this connection we can note that the long-tıme or zero-frequency coupling discussed in Section 2 reflects all the character of a static electric field The effect of molecule B on the selection rules of molecule A is to change its allowed transitions, specifically, if $A$ is centrosymmetric then parity-preserving transitions are permitted This is exactly the same behaviour as results from the application of a static field, as we would expect

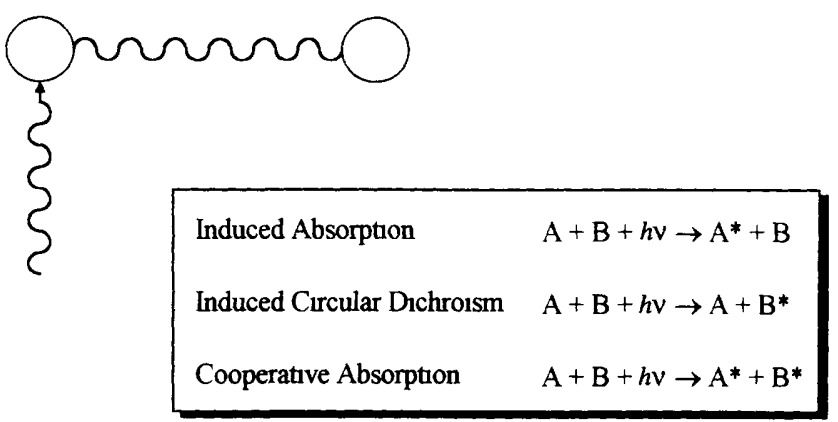

Figure 4 Varıous one-photon bimolecular interactıons

A similar type of bimolecular interaction where one molecule plays a passive role is Induced Circular Dichroism (ICD) It is well known that the absorption of light by chiral molecules is marginally different for left and right circular polarızations, a phenomenon known as natural circular dichroism Molecules which are intrinsically achiral can nonetheless display differential absorption when in the neighbourhood of a chiral molecule see for example recent work on inclusion complexes ${ }^{12}$ The essential feature is that the dichroism is seen at frequencies characteristic of the achiral molecule $\mathbf{B}, \mathbf{A}+\mathbf{B}+h \nu \rightarrow \mathbf{A}+\mathbf{B}^{*}$, and can be distinguished from the response of $A$, the chiral species To develop the theory of ICD, magnetic dipole as well as electric dipole interactions have to be taken into account ${ }^{1314}$ at this level of approximation, chirality can be manifest only where transitions are both electric and magnetic dipole allowed ${ }^{+}$ Once again the interaction between the molecules involved is as represented in Figure 4, and it is quantum interference between this bimolecular interaction and normal photoabsorption by $B$, $\mathbf{B}+h \nu \rightarrow \mathbf{B}^{*}$, that generates the chiral response

\section{Cooperative Absorption}

The process of cooperative absorption is distinguished by the fact that the energy from each photon of the absorbed radiation

+ For dll other processes discussed in this review the electric dipole approximd tion is sufficient other multipolar contributions being generally negligible 
is taken up by two molecular species, $\mathrm{A}+\mathrm{B}+h v \rightarrow \mathrm{A}^{*}+\mathrm{B}^{*}$, again as in Figure 4 . Since both molecules are promoted to excited states, the energy which is transferred from the primary absorber $A$ is necessarily the full excitation energy for the secondary recipient $\mathbf{B}$. This is an effect first seen in molecular gases by bimolecular combination frequency absorption in the infrared. ${ }^{15}$ In such cases, the two radiation-molecule interactions at $\mathrm{A}$ lead to selection rules of the kind normally associated with Raman-active vibrations, whilst $\mathrm{B}$ follows the normal infrared selection rules. This gives the interesting feature that absorption can appear to take place outside the normal absorption bands of either molecule. Also, final states may be reached in molecule A that differ from those normally allowed - if the molecule possesses a high degree of symmetry. ${ }^{16}$ Such effects are not restricted to the infrared and may also be identified in UV/ visible spectroscopy. Three separate groups ${ }^{17-19}$ have derived results using molecular QED, explicitly showing the various mechanisms that have to be taken into account.

One interesting development is the recent discovery that the intensity of cooperative absorption may be substantially enhanced if one of the molecules is strongly polar; ${ }^{20}$ this may be regarded as reflecting its electromagnetic influence effectively lowering the symmetry of its neighbour. It is interesting to consider the operation of this mechanism where the two molecular species concerned lie on opposite sides of a phase boundary or interface, as for example with two immiscible liquids, one polar and the other non-polar. Under such circumstances it has been shown that if the polar phase has a component with a strong intramolecular charge transfer transition, it can display significant absorption outside its normal absorption band. Such behaviour can uniquely characterize the interface, as shown in Figure 5 . Since the effect proves to be quadratically dependent upon the difference in the dipole moments of the polar species' ground and excited states, molecules with a large internal charge separation, as for example many extensively conjugated organic structures, will be particularly suitable for experimental study.

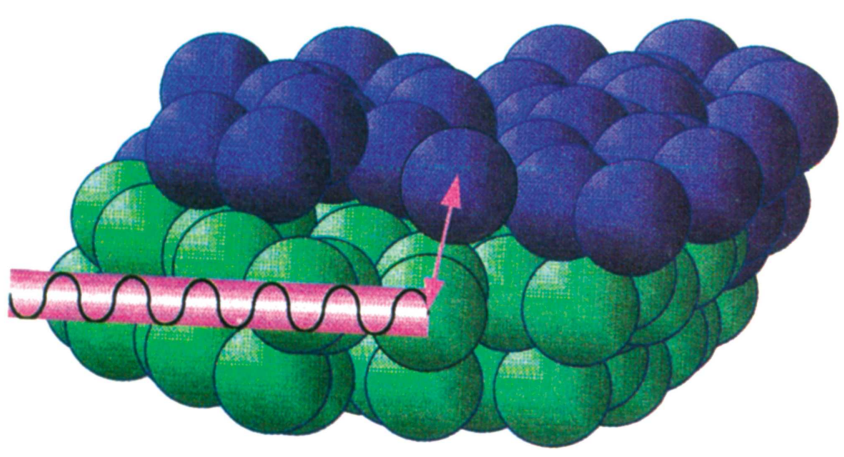

Figure 5 Cooperative absorption at a liquid-liquid interface.

\section{Fluorescence Migration}

The fundamental process that is of interest here is one in which 'donor' molecules A, initially excited on irradiation with light, transfer their energy to 'acceptor' species B which then generate a fluorescence signal. Since under other circumstances fluorescence might be emitted by the initially excited donor species, we employ the concise term 'fluorescence migration' to denote the bimolecular interaction. Here, however, the range of wavelengths over which emission is detected is taken to exclude direct fluorescence from A. Equally, it is assumed that the irradiation wavelength is such that absorption occurs only at A.

This is a process that has been the subject of a great deal of theoretical and experimental research, and where the seminal results of early workers such as Förster ${ }^{11}$ and Perrin ${ }^{21}$ have been widely used, and in some cases modified to further explain the many experimental observations. Specifically, the known rangedependence of the transfer rate can employed as a "spectroscopic ruler' to determine separations of chromophore units, a technique that has found important applications in connection with biological structures. This, for example, has recently found use in the quantitative microscopic imaging of animal cell components. ${ }^{22}$ In general, the polarization of the migrant fluorescence also carries information both on the dynamics of the energy transfer process, and on the spatial correlations of the fluorophores, features that are utilized in studying the biophysical mechanisms of energy transfer in photosynthetic and other structures.

In the representation of fluorescence migration shown as Figure 6 , the migration stage within the parenthesis of the chemical equation represents the energy transfer, which may be either radiative or non-radiative depending upon molecular separation. The mechanism for the intramolecular decay, such as depicted by a modified Jablonski diagram in Figure 7, need not concern us - it plays a part neither in the coupling with the input radiation nor in the fluorescence emission. Fourth-order perturbation theory is used to formulate the whole process from the initial absorption to the detected doubly Stokes-shifted signal. ${ }^{+}$

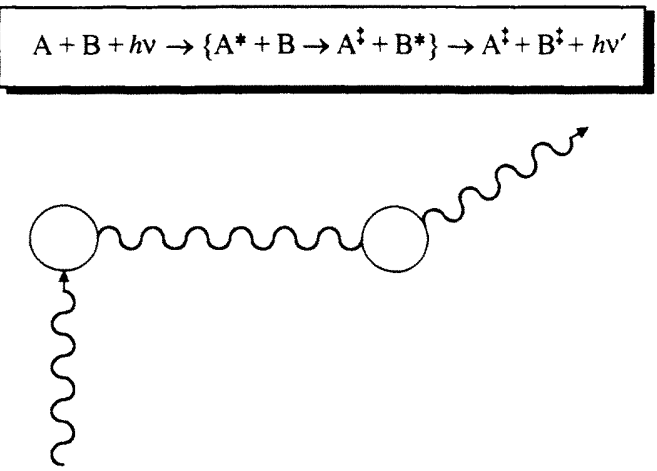

Figure 6 Fluorescence migration. In the equation the double dagger differentiates vibrationally excited states from electronically excited states denoted by asterisks.

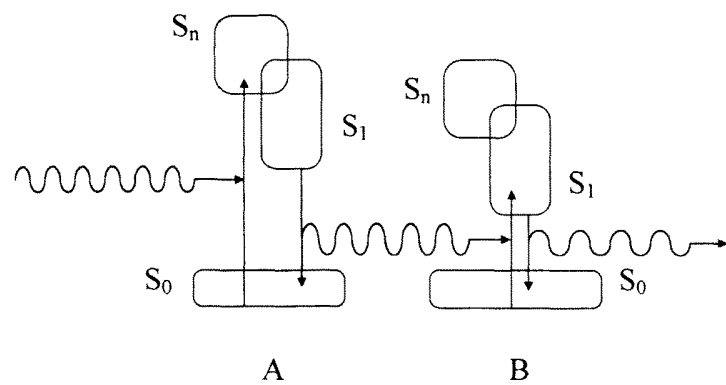

Figure 7 Modified Jablonski diagram showing the essential energetics of fluorescence migration; $S_{0}$ represents the ground electronic state and its associated manifold; $S_{1}, S_{n}$ are higher electronic states.

The polarization parameter commonly measured in fluorescence studies is the polarization anisotropy $r$, obtained by irradiating the sample with plane polarized light. Essentially this parameter represents the difference in the polarization components of the detected light parallel and perpendicular to that of the incident beam, normalized by reference to the net emission. As such it may be defined for each wavelength in the emission spectrum as follows;

$$
r=\frac{\Gamma_{1}-\Gamma_{\perp}}{\Gamma_{1}+2 \Gamma_{\perp}}
$$

Two cases are of particular interest. One is where the participating chromophores $\mathrm{A}$ and $\mathrm{B}$ are part of a larger relatively rigid

+ The term 'doubly Stokes-shifted' signifies that the radiation suffers two consecutive shifts in frequency, one at each of the participating molecules. 
structure, as for example in a protein, and therefore have a strong orientational correlation Here, if $\theta$ denotes the angle between the transition dipole moments for the donor absorption and the acceptor fluorescence, then the following (Perrin) result ensues, ${ }^{21}$

$$
r=\left(3 \cos ^{2} \theta-1\right) / 5
$$

Departures from this behaviour are important indicators of dynamical interplay between intra- and inter-chromophore relaxation, in particular where the possibilities of fluorescence by $\mathrm{A}$ and absorption by $\mathrm{B}$ have also to be entertained ${ }^{23}{ }^{27}$ In the limit where relaxation via intra-chromophore processes is much faster than inter-chromophore energy transfer, the two chromophores act as essentially independent units

The second scenario of interest concerns systems in which the two moieties are structurally uncorrelated ( $l e$ they are randomly oriented or freely rotatıng) Here, it has been shown that under conditions where the rate of molecular rotation is negligible on the fluorescence timescale (as is frequently the case for large polyatomic species) and the transition dipole moments within each molecule for absorption and emission are parallel, then the short- and long-range results for the fluorescence anisotropy are given by, ${ }^{28} 29$

$$
r_{1}=\left\{\begin{array}{l}
\frac{2}{125}=\frac{r_{0}}{25} \text { (short-range) } \\
\frac{14}{125}=\frac{7 r_{0}}{25} \text { (long-range) }
\end{array}\right\}
$$

The parameters $r_{1}$ and $r_{0}$ here denote anisotropy with and without energy transfer between chromophores, respectively The two results show a pronounced difference in the radiative and non-radiative results It is a hallmark of the unified theory of energy transfer that it makes it possible to demonstrate smooth progression from one limiting result to the other as intermolecular distance increases

\section{Two-centre Raman Scattering}

An energy transfer process with many features similar to fluorescence migration is bimolecular Raman scattering, where inc1dent light undergoes Raman scattering by two molecules $\mathrm{A}$ and B, each of which experiences an uptake (or, conceivably a loss) of energy The main difference lies in the fact that, using optical frequencies well away from any absorption bands, the incidence and emergence of radiation at both $\mathrm{A}$ and $\mathrm{B}$ is essentially instantaneous Such two-centre Raman scattering has been the subject of relatively few studies, undoubtedly reflectıng its experimental difficulties, ${ }^{30}$ however it has recently been suggested that there is a possibility of distinguishing bimolecular from conventional Raman signals by their anomalously high depolarization ratios 29

The selection rules for the bimolecular Raman process have to be considered carefully to distınguish the two types of interaction that may occur, illustrated in Figure 8 One obvious possibility, shown in Figure 8(a), is that the incident radiation undergoes successive Raman scattering events at A and B, initially impinging on A but with the detected Raman signal emerging from B In this sequential process, as two photon events are involved for each molecule, the selection rules for each participant are exactly as for conventional Raman transitions A second possibility, Figure 8(b), is where A both intercepts the incident and generates the signal radiation, their energy mismatch being transferred to B As this type of interaction has an odd number of photon interactions at each site, three at the scattering centre and one at the second molecule, it has quite different selection rules, specifically, hyper-Raman selection rules apply to $A$, and infrared selection rules to $B$

The change in optical polarization that accompanies Raman scattering is commonly measured in terms of the depolarization ratio $\rho$, defined as, $\rho=\Gamma_{L} / \Gamma_{\mid}$, where $\Gamma$ represents Raman
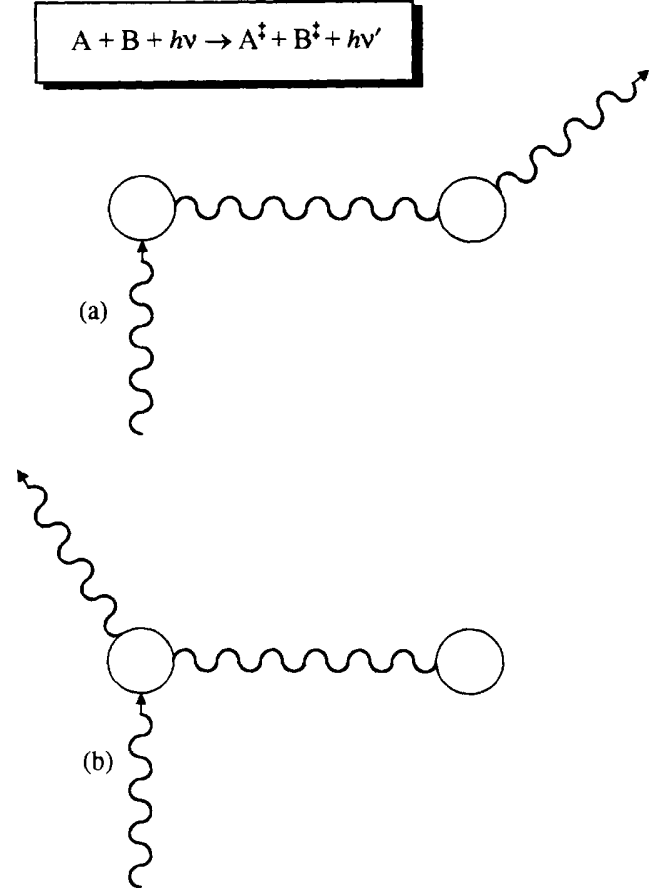

Figure 8 The two mechanısms for bimolecular Raman scatterıng

scattering with the same plane polarization as the incident radiation, and $\Gamma_{\perp}$ denotes scattering polarized at right angles to the incident polarization The bimolecular depolarization ratio $\rho_{1}$ is similarly defined for Raman signals associated with pairwise energy transfer between molecules Results for $\rho_{1}$ have been calculated for the long and short range limits of radiative and non-radiatıve energy transfer, accommodatıng all possible conditions It has been found that the results yield a depolarization ratio exceeding the normal off-resonant limit of $3 / 4$, both at and away from resonance, allowing an experimental parameter through which it may be possible to detect the bimolecular process For example sequential Raman scattering involving depolarized bands in both $\mathrm{A}$ and $\mathrm{B}$ leads to almost complete demolition of the polarization, detaled calculations show that here $\rho_{1}$ differs only marginally from unity (in fact by almost exactly $03 \%$ ) for molecules in close proximity 29

\section{Bimolecular Two-Photon Absorption}

The interest in this type of bimolecular interaction, $\mathrm{A}+\mathrm{B}$ $+2 h \nu \rightarrow \mathrm{A}^{*}+\mathrm{B}^{*}$, hes in the possibility of synergistically exciting two different molecular species through absorption of radiation at a frequency that neither species would absorb on its own As shown in the energetıcs scheme for this process, Figure 9 , it can be assumed that nether A nor B absorbs either at frequency $v$ or $2 v$, indeed the absorbed frequency is necessarlly a mean of

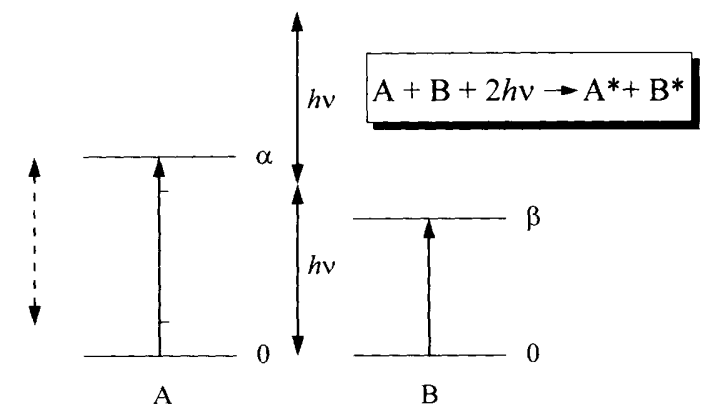

Figure 9 Schematic energetics for bimolecular two-photon absorption Dotted lines indicate where the possible presence of other molecular energy levels affords opportunities for resonance enhancement 
absorption frequencies for the two molecular participants As lllustrated in Figure 10 there are once again two mechanisms that can operate, according to whether the two absorbed photons impinge on different centres, (a), or both impinge on one, (b), in previous studies these have been referred to as cooperative and distributive mechanisms, ${ }^{31} 32$ respectively Since they have rather different features, we shall deal with each mechanism in turn

$$
\mathrm{A}+\mathrm{B}+2 h v \rightarrow \mathrm{A}^{*}+\mathrm{B}^{*}
$$
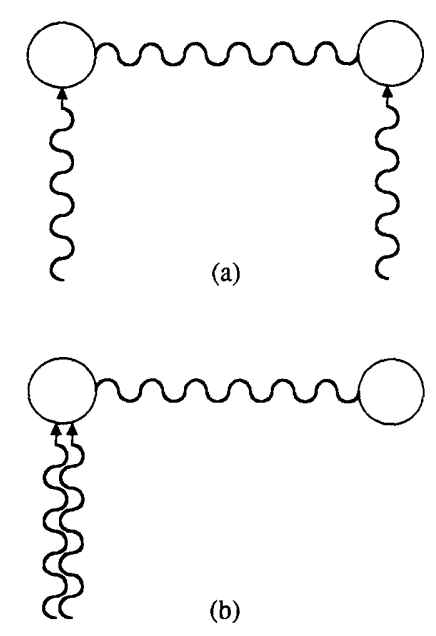

Figure 10 The two mechanisms for bimolecular two-photon absorption

Consider first mechanism (a) When molecule A, initially in its ground state, undergoes a transition to an excited state a through absorption of one quantum of light with a freqency $\nu$, then since $\nu$ is off-resonant with respect to the transition frequency there is a mismatch in energy by an amount $\delta E=h v-E_{\mathrm{a}}$ (assuming that energies are referred to the ground state as zero) A suitable mechanism for compensation of the energy mismatch is provided by the cooperative excitation of molecule $B$, through absorption of another photon with the same frequency $\nu$, to an excited state $\beta$ with energy $E_{\beta}=h \nu-\delta E$ Thereby the overall energy conservation $E_{\alpha}+E_{\beta}=2 h \nu$ is satisfied

Here, the Uncertainty Principle shows that it is impossible to constrain conservation of energy over a timescale less than $\tau \sim\left(2 \pi \nu-E_{a} / \hbar\right)^{-1}$, the transitions are therefore allowed provided the local energy mismatch persists for a time not exceeding $\tau$ The implication is that for the cooperative process to occur, the two molecules should be separated by a distance broadly bounded by $c \tau$, which might even with visible frequency excitation be a distance comparable to an infrared wavelength Over larger distances the process acquires the characteristics of electronic Raman scattering at A followed by a separate process of two-photon absorption at B The selection rules for this twophoton cooperative absorption show that we must have a return to the parity of the initial state by both molecules in order for this type of excitation to take place

The distributive mechanısm, Figure 10(b), involves absorption of two photons by molecule A, with coupling of the excess energy to the second absorber B As with conventional twophoton absorption, there is no need for A to possess an energy level corresponding to the photon energy, and thus no identifiable intermediate state is populated Here energy conservation should be realised within a time $\tau \sim\left(E_{\beta} / \hbar\right)^{-1}$, corresponding to a range of intermolecular distances roughly bounded by the wavelength of the radiation The long-range behaviour is then identifiable with electronic hyper-Raman scatterıng at A followed by conventional single-photon absorption at B Here the transitions of both molecules are associated with a change in parity For both mechanisms, more detarled selection rules have been derived for molecules of arbitrary symmetry 16

Bimolecular two-photon absorption is undoubtedly a weak effect that requires intense laser radiation for its observation Nonetheless it has been experimentally characterized in a variety of media, mostly in connection with charge-transfer transitions involving molecular halogens and inert gases ${ }^{33}$ The counterpart mean-frequency process, in which two molecules of the same chemical species are excited in a two-beam experiment by photons of differing frequency has so far received only theoret1cal treatment, though it presents unique opportunities for exploiting resonance enhancement

\section{Quantum Effects in the Absorption of Broadband Radiation}

As a final example of bimolecular photophysics, we can look at a process that may significantly change the appearance of what are ostensibly normal single-photon absorption spectra Though the effects to be described will only be manifest at very high intensities, they are distinct from the saturation processes that can also lead to spectral change The principal issue here is an important but generally unquestioned tenet of absorption spectroscopy with broadband sources, that spectra obtained by scanning the wavelength of incident light are identical to those obtained with all wavelengths simultaneously present This is, of course, the basis of Fourier Transform spectroscopy Nonetheless it is an assumption that at high intensities fails

The mechanism at work here is none other than two-photon bimolecular absorption Consider a system of identical molecules irradiated by intense broadband light Two molecules of the same species in close proximity can absorb any pair of photons whose mean energy equals that of an upward trans1tion Under sufficiently intense illumination there will in fact be a large number of such photon parrs that can do the job Once more the energy mismatch propagates across between the molecules involved in order for energy finally to be conserved in each one The main effect of such processes is that the normal Beer-Lambert law has to be modified to the following form

$$
-\frac{\mathrm{d} I(\nu, x)}{\mathrm{d} x} \propto\left[I(\nu, x)+\kappa \int K(\nu, \zeta) I(\nu+\zeta, x) I(v-\zeta, x) \mathrm{d} \zeta\right],(8)
$$

where for a given frequency $\nu$ the familar exponential decay of intensity $I$ with distance $x$ results if only the leading term on the right is present The correction term given by the integral represents a convoluted response spanning all frequencies present in the incident light, $\zeta$ signifying the correlated frequency mismatches of the two photons, and $K$ characterizing the material response The most obvious manifestation of this addition to the linear term is a change in spectral linewidths ${ }^{34}$

An apparently ideal source for observations of this effect is the ultrafast supercontinuum generated on passage of mode-locked laser light through a variety of media, including nothing more sophisticated than a beaker of water Here we have broadband emission that can cover several hundred nanometres of wavelength in the visible, at intensity levels that should be adequate for cooperative excitation process to occur Under such conditions, then even with an ideal dispersive spectrometer, spectra recorded with a monochromator placed between the broadband source and the sample can be expected to differ from spectra recorded with the monochromator between the sample and detector (since in the former case the sample will be illuminated only by the narrow band of radiation transmitted by the exit slit of the monochromator) This set up should enable anomalous absorption to be observed and discrimınated from any competing saturation To our knowledge, the task of experimentally identifying this bimolecular effect is a challenge yet to be accepted

\section{Conclusion}

In this review we have presented a brief survey of a variety of 
bimolecular photophysical interactıons where electromagnetıc coupling plays a central role In particular, we have attempted to 1llustrate the use of quantum electrodynamics in conceptualizing and interpreting these processes For example, it is only by the use of such methods that 'radiationless' and radiative coupling can be shown not to comprise competitive mechanısms for energy transfer

We have specifically dealt with the interactions of a two-body system Since coupling extends beyond nearest neighbours, the possibility of trimolecular or even higher order processes can conceivably arise, ${ }^{35}$ though at present experimental observations appear fully consistent with pair coupling The influence of the electronic fields of intervening molecules can also be addressed, though at cost of considerably increased theoretical complexity Nonetheless, additional features attributable to attenuation and refraction can then be identified, facilitating more realistic modelling of bulk systems ${ }^{8} W_{1}$ th these elements of theory now well in place, there is ample scope for further explorations and discoveries of new applications in the field of bimolecular photophysics

Acknowledgments We are pleased to acknowledge helpful comments from Professor Norman Sheppard FRS and Dr Andrey A Demidov Our research is supported by grants from the Engineering and Physical Sciences Research Council

\section{References}

I E Fermı, 'Nuclear Physics', University of Chicago Press, Chicago, 1950

2 J S Avery, Proc Phys Soc, 1966, 88, 1

3 L Gomberoff and E A Power, Proc Phys Soc, 1966, 88, 281

4 D L Andrews, Chem Phys, 1989, 135, 195
5 E A Power and T Thirunamachandran, Phys Rev A, 1983, 28, 2671

6 D L Andrews and B S Sherborne, $J$ Chem Phys, 1987, 86, 4011

7 D L Andrews and G Juzeliūnas, $J$ Chem Phys, 1992, 96, 6606

$8 \mathrm{G}$ Juzelıủnas and D L Andrews, Phys Rev B, 1994, 49,875

9 H B G Casımir and D Polder, Phys Rev, 1948, 73, 360

10 G D Scholes, A H A Clayton, and K P Ghiggino, J Chem Phys, $1992,97,7405$

11 J T Forster, $Z$ Naturforsch, 1949, 4a, 321

12 G Marconı, S Montı, B Mayer, and G Kohler, $J$ Phys Chem, 1995, 99, 3943

13 D P Craig, E A Power, and T Thirunamachandran, Proc Roy Soc Lond A, 1976, 348, 19

14 P E Schipper, Chem Phys, 1981, 57, 105

15 J A A Ketelaar, Recl Trav Chim Pays-Bas, 1956, 75, 857

16 D L Andrews and A M Bittner, Chem Phys, 1992, 165, 1

17 E Hudis, Y Ben-Aryeh, and U P Oppenheim, Phys Rev A, 1991, 43, 3631

18 S John and J Wang, Phys Rev B, 1991, 43, 12772

19 G Kweon and L M Lawandy, Phys Rev B, 1994, 49, 4445

20 D L Andrews and P Allcock, Chem Phys Lett, 1994, 231, 206

21 F Perrin, Comptes Rendus, 1925, 180, 581

22 Z Kam, T Volberg, and B Geiger, $J$ Cell $S c t, 1995,108,1051$

23 P A Anfinrud and W S Struve, $J$ Chem Phys, 1987, 91, 5058

24 G Fredrickson, J Chem Phys, 1988, 88, 5291

25 A A Demidov, $J$ Theor Biol, 1994, 170, 355

26 A Matro and J A Cina, $J$ Phys Chem, 1995, 99, 2568

27 A A Demidov and D L Andrews, Chem Phys Lett, 1995, 235. 327

$28 \mathrm{D}$ L Andrews and G Juzelıūnas, $J$ Chem $P h_{y} s, 1991,95,5513$

29 D L Andrews and P Allcock, Chem Phys 1995, in press

$30 \mathrm{D}$ L Andrews and N P Blake, Phys Rev A, 1990, 41, 2547

31 D L Andrews and M J Harlow, $J$ Chem Phys, 1983, 78, 1088

$32 \mathrm{D}$ L Andrews and K P Hopkıns, Adv Chem Phys, 1990, 77, 39

33 M E Fajardo, R Withnall, J Feld, F Okada, W Lawrence, L Wiedeman, and V A Apkarian, Laser Chem, 1988, 9, 1

34 D L Andrews, Phys Rev A, 1988, 38, 5129

35 D P Craig and T Thirunamachandran, Chem Phys 1989, 135, 37 